\title{
Análisis y evolución de la cartografía de zonas inundables en España, desde un marco jurídico de referencia nacional a uno europeo
}

Francisco López-Martínez. Universidad de Murcia, Murcia, España.

RESUMEN | Durante los últimos años, los mapas de extensión de la inundación se han convertido en un elemento fundamental para la gestión preventiva de los riegos de inundación. Aunque a nivel europeo el desarrollo de esta herramienta estuvo impulsado por la Directiva 2007/60/CE, en algunos países como Espańa, donde el impacto de las inundaciones sobre las sociedades ha adquirido un protagonismo primordial, su uso ya había sido previamente implementado. En este contexto, el presente estudio describe las principales características de cada cartografía, la primigenia Directriz Básica y el vigente Sistema Nacional de Cartografía de Zonas Inundables (sNCZI), analizando, además, sus diferencias, deficiencias y puntos de mejora. Considerando todos estos aspectos, el SNCZI optimiza ampliamente los resultados de la Directriz Básica, pues no solo aumenta el número de periodos de retorno estudiados, sino que también mejora la metodología, datos de partida, cartografía disponible y accesibilidad a la misma.

PALABRAS CLAVE | riesgos naturales, ordenamiento territorial, vulnerabilidad.

ABSTRACT | Over the last years, flood hazard maps became a crucial element of flood risk management policies. Although this tool was boosted at European level by the Directive 2007/60/EC, in several countries such as Spain, where floods have had an historical relevance on societies, its implementation has been previously developed. Thus, the present study describes the main characteristics of both cartography, the primitive Basic Guideline and the current SNCZI [National System for Cartography of Flood-Prone Areas, by its acronym in Spanish], in addition, it also analyses their differences, shortcomings and improvement points. Considering these issues, the SNCZI greatly improves the Basic Guidelines results, not only because it increases the number of likely return period studied, but also enhances the methodology, initial data, available cartography and its public accessibility.

KEYWORDS | natural risks, spatial planning, vulnerability. 
En el territorio español, "las inundaciones constituyen el riesgo natural que a lo largo del tiempo ha producido los mayores daños tanto materiales como en pérdida de vidas humanas" (Real Decreto 903/2010²). De hecho, según datos del Consorcio de Compensación de Seguros ${ }^{2}$ (2017), durante las tres últimas décadas, ${ }^{3}$ las inundaciones han sido responsables de 4.9 trillones de euros en pérdidas económicas y 231 fallecidos. El desmesurado volumen de estas cifras, cuyos registros a lo largo de los últimos años no han hecho más que aumentar (Consorcio de Compensación de Seguros, 2017), ha dado lugar a una progresiva e ingente preocupación por parte del Gobierno Nacional en lo que a la gestión preventiva de los riegos de inundación, así como sus dańos derivados, se refiere. Por este motivo, el actual marco jurídico nacional y autonómico está conformado por un amplio entramado de normativas, bien sean de carácter sectorial (por ejemplo, agua, costas, medioambiente o industria) o específico (usos del suelo y urbanismo), encaminadas a reducir la exposición de las sociedades a los riesgos de inundación.

Tradicionalmente, esta situación ha sido contrarrestada a través de ingentes y costosas medidas estructurales que han contemplado desde los sistemas de retención (por ejemplo, presas, tanques de tormenta), pasando por los de drenaje (por ejemplo, alcantarillado, zanjas), hasta finalizar en los de protección (por ejemplo, diques, encauzamiento, aislamientos). Sin embargo, además de los efectos directa o indirectamente asociados a estas medidas, que posiblemente no habían sido cuantificados en su totalidad durante el proceso de redacción de las mismas (por ejemplo, gastos asociados, impacto ambiental o costes de mantenimiento), diversos estudios han puesto de manifiesto que sus resultados, tal y como ya demostró la denominada "paradoja hidráulica" expuesta por White (1958) tras la aprobación de la ambiciosa "Flood Control Act (1936)" en Estados Unidos, no siempre han sido los deseados. En efecto, la falsa sensación de seguridad derivada de este tipo de medidas ingenieriles, lejos de alcanzar su cometido inicial, se ha materializado en un aumento directo de la exposición al riesgo tras haberse invadido, transformado y ocupado las zonas inundables. Esta circunstancia, ampliamente desarrollada en la literatura científica, ha sido definida según el autor consultado como "escalator effect" (Parker, 1995), "levée effect" (Lane, Landström, \& Whatmore, 2011) o "paradoja del desarrollo seguro" (Burby, 2006).

Por consiguiente, demostrada la ineficiencia de las infraestructuras hidráulicas como medida paliativa ante los efectos -infortunadamente cada vez más catastróficos- de las inundaciones, el siguiente paso consistió en integrar las dinámicas sociales como una parte indispensable de las políticas encargadas de la gestión de riegos. En esta nueva perspectiva de adaptación ambiental, la ordenación territorial emergió como una de las medidas preventivas no estructurales clave para condicionar y limitar la exposición de las sociedades ante los riesgos de inundación.

1 Real Decreto 903/2010, de 9 de julio, de evaluación y gestión de riesgos de inundación.

2 El Consorcio de Compensación de Seguros está configurado como una entidad pública empresarial encargada de cubrir, en régimen de compensación económica, a los ciudadanos espańoles afectados por siniestros ocurridos tanto en territorio nacional como en el extranjero.

3 Considerando el periodo comprendido desde 1987 a 2017. 
Aunque las distintas normativas, competencias e instrumentos de ordenación territorial dependen de la organización político-administrativa interna de cada país, diversas han sido los programas, reglamentaciones o planes publicados que se han encargado de imbricar los riesgos de inundación como un criterio indispensable durante el proceso de redacción de las políticas territoriales.

Desde un punto de vista jurídico, hay que remontarse hasta 1995 para encontrar, dentro del Estado español, una de las primigenias y vanguardistas normativas encargadas de la gestión de los riegos de inundación a través de medidas no estructurales: la Directriz Básica de Planificación de Protección Civil ante el Riesgo de Inundaciones (en adelante Directriz Básica). Aunque con carácter legislativo propio, la Directriz Básica surgió como respuesta al anterior Real Decreto 407/1992, ${ }^{4}$ reglamento relacionado con los servicios de protección civil y que establecía la obligatoriedad de elaborar una serie de "Planes Especiales" relacionados con diversos riegos, tanto de origen natural (por ejemplo, vulcanismo, seísmos o incendios forestales) como inducido (por ejemplo, emergencias nucleares, conflictos bélicos o transporte de cargas peligrosas). No obstante, e independientemente de su origen, la Directriz Básica representa el primer texto legal de ámbito nacional encargado de establecer medidas de organización y procedimiento de actuación ante los riesgos de inundación, así como sus posibles efectos asociados, pues no debe olvidarse que, tal y como indica el Preámbulo de la misma, "las inundaciones son el fenómeno natural que con mayor frecuencia se manifiesta dando lugar a situaciones de grave riesgo colectivo o catástrofe". Con la intención de neutralizar esta dura realidad, el articulado de la Directriz Básica decretaba que cada comunidad autónoma, además del Gobierno Nacional, debía desarrollar y aprobar diversos planes capaces de garantizar una respuesta eficaz de la administración ante estados de emergencia (apartado 3.3 y 3.4 , respectivamente).

Entre los múltiples aspectos que debían contener dichos planes (estructura organizativa, recursos humanos y materiales, sistemas y medios de información, etcétera), la Directriz Básica indicaba que las comunidades autónomas tenían la obligación de identificar, representar y clasificar cartográficamente las áreas potencialmente inundables, además de la posible superficie afectada por sus fenómenos asociados. En este sentido, el legislador nacional estableció diferentes superficies inundables, categorizadas en función del periodo de retorno ( $T$, expresado en ańos), así como zonas de riesgo, definidas según las características de las avenidas y tipología de la población afectada (núcleos urbanos, viviendas aisladas, comercios, industrias, etcétera). Posteriormente, todos estos aspectos autonómicos debían ser integrados en un "Plan Estatal", cuyo objetivo consistía en establecer la organización y los procedimientos de actuación de aquellos servicios del Estado y, en su caso, de otras entidades públicas y privadas, que fueren necesarios para garantizar una repuesta eficaz ante los diferentes tipos de inundaciones que puedan producirse o afectar al territorio espańol (apartado 3.3 y sucesivos). 
Atendiendo a los diversos periodos de retorno definidos, las áreas potencialmente inundables debían quedar clasificadas a nivel autonómico como: i) frecuente $(\mathrm{T}<50)$, ii) ocasional $(50<\mathrm{T}<100)$ y iii) excepcional $(100<\mathrm{T}<500)$. Por otro lado, respecto a las zonas de riesgo, también existía una categorización en subzonas (A-1, A-2, A-3, B y C), determinadas según la combinación entre la anterior clasificación de áreas inundables (respectivamente, de mayor a menor probabilidad) y la tipología de bienes inmuebles ubicados en las mismas. ${ }^{5}$ En conjunto, toda esta catalogación y categorización de los riesgos de inundación debía estar cartográficamente plasmada en los distintos planes autonómicos, configurándose, tal y como establecía el Acuerdo Segundo de la Directriz Básica, como la cartografía oficial de consulta por parte de las administraciones encargadas de la ordenación territorial y urbanística durante el procedimiento de clasificación y calificación (Beltrán Aguirre, 20066 de los usos del suelo.

Sin embargo, a pesar de que la Directriz Básica introducía preceptos legales y herramientas tan novedosos para la época como la categorización y representación cartográfica de los riesgos de inundación, desafortunadamente su impacto no alcanzó los efectos deseados. Efectivamente, cinco años después de su publicación, solo tres comunidades autónomas (Madrid en 1997, País Vasco en 1999 y la Comunidad Valenciana en 1999) habían elaborado, aprobado y homologado sus respectivos Planes Especiales ante Inundaciones, cifra que solo se ampliaría en cuatro más en el siguiente lustro (Navarra y Galicia en 2002, Andalucía en 2004 y Baleares en 2004). Esta negligente situación de retraso normativo adquirió especial relevancia en algunas de las comunidades del litoral mediterráneo peninsular (Cataluña, Región de Murcia y Andalucía), pues la histórica e inusual concentración de personas y bienes en dicha zona la convirtieron, además del motor de crecimiento inmobiliario de Espańa junto con las áreas metropolitanas (Gonzáles Reverté, 2008), en una de las más castigadas por los riesgos de inundación (Nájera Ibáñez \& Espejo Gil, 2017). En consecuencia, aparte del elevado riesgo que suponía para la población la indefinición de las zonas inundables y su correspondiente utilización durante el proceso de planificación territorial, esta demora legislativa también acarreó un notorio retraso en la publicación del Plan Nacional, cuyo texto no sería aprobado sino hasta el año 2011.

Tras dos décadas de vigencia de la Directriz Básica, el Parlamento Europeo, preocupado por el posible aumento en número e impacto de las inundaciones, publicó en 2007 una directiva encaminada a evaluar y gestionar sus riesgos asociados, la Directiva 2007/60/CE. ${ }^{7}$ Entre otros novedosos aspectos, como la obligatoriedad de

5 Más que con las características constructivas, este aspecto estaba íntimamente relacionado con la densidad poblacional del asentamiento; por ejemplo, núcleos urbanos, viviendas aisladas, instalaciones comerciales, etcétera.

6 Según este autor, mientras "clasificar" el suelo consiste en asignar su futuro destino urbanístico (urbano, urbanizable y no urbanizable), su "clasificación” está relacionada con determinar y definir los usos (por ejemplo, residencial, comercial, dotacional) e intensidades (por ejemplo, vivienda unifamiliar aislada, colectiva, número de alturas) del mismo.

7 Directiva 2007/60/CE del Parlamento Europeo y del Consejo de 23 de octubre de 2007 relativa a la evaluación y gestión de los riegos de inundación. 
establecer un marco supranacional para la evaluación y gestión de los riegos de inundación (art. 1), la Directiva fue el primer texto jurídico comunitario encargado de conceder a la ordenación territorial una posición destacada como primer instrumento defensivo para reducir la exposición ante los riegos de origen natural. Para alcanzar dicho cometido, la Directiva encomendó a los Estados miembros que sobre cada demarcación hidrográfica desarrollaran diversos mapas de extensión de la inundación, mapas que deberían quedar configurados según los siguientes periodos de retorno: i) baja probabilidad o escenario de eventos extremos $(\mathrm{T}<10)$, ii) frecuente $(10<\mathrm{T}<50)$, iii) media u ocasional $(50<\mathrm{T}<100)$, y iv) baja o excepcional $(\mathrm{T}<500)$.

Aunque todos los preceptos de la Directiva fueron traspuestos al ordenamiento jurídico interno del país a través del Real Decreto 903/2010, previamente el Gobierno Nacional había incorporado, a través del Real Decreto 9/2008, ${ }^{8}$ algunas de sus disposiciones y herramientas, como los estudios de inundabilidad o los mapas de extensión de la inundación. A este respecto, el segundo Real Decreto contemplaba la elaboración del Sistema Nacional de Cartografía de Zonas Inundables (sNCZI), instrumento de apoyo nacional desarrollado para la gestión del espacio fluvial, la prevención de riesgos de inundación, la planificación territorial y la transparencia administrativa. El actual SNCZI ha desplazado las anteriores áreas inundables establecidas por la Directriz Básica, convirtiéndose así en la cartografía legal de consulta (art. 10.3), tras aumentar tanto la delimitación y precisión espacial, como el número de escenarios posibles ante los riesgos de inundación en: i) alta (T10), ii) frecuente (T50), iii) medio u ocasional (T100), y iv) bajo o excepcional (T500). Asimismo, otras de las grandes novedades recogidas en el SNCZI son la multitud de formatos digitales utilizados para difundir y poner a disposición de la ciudadanía toda la información relativa a las áreas inundables, pudiendo acceder a la misma a través de un geoportal alojado en la propia web del Ministerio para la Transición Ecológica ${ }^{9}$ e, incluso, integrándola perfectamente en un SIG a través de un servidor $w m s^{10}$ o por la descarga directa de todos los datos en extensión shapefile. ${ }^{11}$

Como resultado de la mejora en los datos de partida (por ejemplo, resolución del modelo digital del terreno, registros pluviométricos o transformaciones antrópicas del terreno), así como los avances tecnológicos (hardware y software), el sNCZI ha reducido diversas limitaciones contenidas en la Directriz Básica, especialmente en lo que a extensión de las zonas inundables se refiere (figura 1). Además, la Directiva también se encargó de impulsar satisfactoriamente la consideración de esta poderosa herramienta cartográfica dentro de los procesos de ordenación territorial y urbanística, intentando así evitar la desidia en que cayó la Directriz Básica desde su publicación. Sin embargo, a pesar de los enormes avances introducidos, el vigente SNCZI se enfrenta a tres cuestiones principales que deben ser solucionadas con la mayor

8 Real Decreto 9/2008, de 11 de enero, por el que se modifica el Reglamento del Dominio Público Hidráulico.

9 https://sig.miteco.es/snczi

10 https://www.miteco.gob.es/wms/agua

11 https://www.miteco.gob.es/descargas/agua/snczi 
celeridad posible: i) la escasa superficie cartografiada, ii) la extensión temporal de los registros pluviométricos y, iii) su continua actualización.
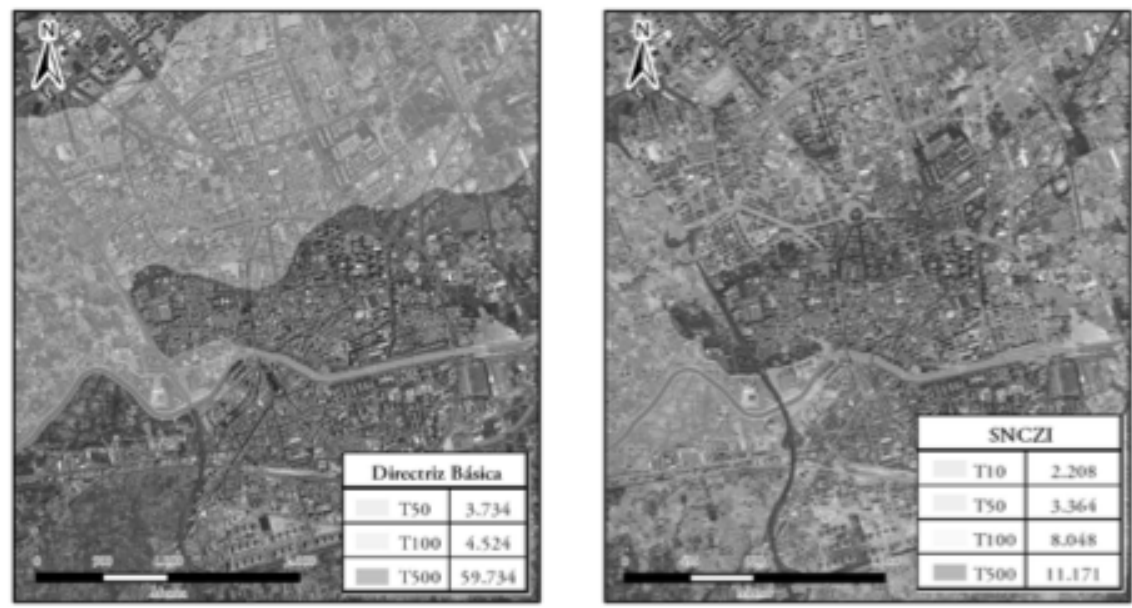

FIGURA I | Diferencias en la extensión de las zonas inundables

(expresadas en hectáreas) según la Directriz Básica (izquierda) y el

Sistema Nacional de Cartografía de Zonas Inundables (derecha) para el municipio de Murcia (Región de Murcia)

FUENTE: ELABORACIÓN PROPIA

En primer lugar, considerando a la última versión cartográfica disponible en la página web del Ministerio para la Transición Ecológica, ${ }^{12}$ los tramos estudiados corresponden a $21.125 \mathrm{~km}$ para T10, $26.014 \mathrm{~km}$ para T100 y $27.381 \mathrm{~km}$ para T500, es decir, una media de aproximadamente el 30\% de los cauces principales. Estas cifras suponen un estadio bastante retrasado con respecto a las disposiciones establecidas en la Directiva y el Real Decreto 903/2010, pues, según los mismos, tanto los mapas de peligrosidad de inundaciones como los mapas de riesgos de inundación debían estar finalizados, a más tardar, el 22 de diciembre de 2013 (art. 6.8 y 10.6, respectivamente). En segundo lugar, todavía existen problemas relacionados con los datos pluviométricos utilizados para el cálculo de los distintos periodos de retorno, ya que la ausencia de registros lo suficientemente extensos de precipitación ha dado lugar al cálculo de eventos extremos (T500) a partir de datos empíricos cuya extensión raramente supera los cien años, o no están suficientemente validados. Por consiguiente, además de asumir un comportamiento "repetitivo" de la naturaleza, es muy posible que la vigente cartografía haya subestimado la auténtica superficie inundable, especialmente para aquellos eventos menos frecuente (T100 y T500). En tercer y último lugar, debido a que las actividades humanas están continuamente modificando la morfología fluvial, el régimen de caudales o los procesos de sellado en la cuenca vertiente hasta prácticamente su completa desnaturalización, 
la extensión, calado y peligrosidad de las zonas inundables ha sido profundamente alterada. Dicho contexto cobra especial relevancia tras la pertinente modelización de los cauces, situación que lleva emparejada una inviable y prácticamente continua actualización cartográfica de las zonas inundables (Olcina Cantos, 2012) capaz de recoger las alteraciones introducidas tanto en su extensión espacial como niveles de peligrosidad.

Como conclusión, las enormes mejoras técnicas, procedimentales, espaciales y preceptivos introducidos por la Directiva respecto a su predecesora a nivel nacional, la Directriz Básica, han convertido al SNCZI en una potente herramienta cartográfica fácilmente consultable e integrable dentro del proceso de ordenación territorial. Sin embargo, a pesar de la dilatada progresión y versatilidad que lo ha caracterizado a lo largo de los últimos años, el SNCZI se enfrenta actualmente a una serie de deficiencias que deben ser rápidamente contrarrestadas; entre otras, una mayor consideración por parte de los gestores territoriales, evitando así la aparición de la denominada "paradoja del gobierno local" (Burby, 2006). Por consiguiente, dotar a las administraciones competentes, esencialmente a las encomendadas de la gestión hidrológica (en España, las confederaciones hidrográficas), de los recursos económicos, humanos e instrumentales necesarios, se convierte en una tarea estratégica y fundamental para optimizar, a corto y mediano plazo, la eficiencia de esta herramienta como medida preventiva para la gestión de riesgos de inundación. En último lugar, tampoco deben caer en el olvido los planes autonómicos ante el riesgo de inundación, pues aunque han visto desplazadas sus delimitaciones cartográficas por el actual sNCZI, el carácter organizativo establecido en los mismos ante situaciones de emergencia sigue vigente en su totalidad, lo que también hace necesaria la actualización de sus protocolos de actuación ante nuevos eventos, especialmente considerando las posibles alteraciones introducidas en los patrones de inundación como consecuencia del cambio climático y de las actividades humanas.

\section{Referencias bibliográficas}

Beltrán Aguirre, J. L. (2006). Clasificación, categorización y calificación del suelo en la legislación autonómica comparada. Revista Jurídica de Navarra, (41), 81-12.

Burby, R. J. (2006). Hurricane Katrina and the paradoxes of government disaster policy: bringing about wise governmental decisions for hazardous areas. The Annals of the American Academy of Political and Social Science, 604(1), 171-191. https://doi. org/10.1177\%2F0002716205284676

Consorcio de Compensación de Seguros. (2017). Estadística. Riesgos extraordinarios. Serie 19712017. Ministerio de Economía, Industria y Competitividad, Gobierno de España. Madrid, España, 149 pp.

Directiva 2007/60/CE del Parlamento Europeo y del Consejo, de 23 de octubre de 2007, relativa a la evaluación y gestión de los riesgos de inundación. Diario Oficial de la Unión Europea, Luxemburgo. oJ, L 288, 27-34. 
Directriz Básica de Planificación de Protección Civil ante el Riesgo de Inundaciones (1995). Resolución de 31 de enero de 1995, de la Secretaría de Estado de Interior. Boletín Oficial del Estado, Madrid, España. воE-A-1995-3865.

González Reverté, F. (2008). El papel de los destinos turísticos en la transformación sociodemográfica del litoral mediterráneo español. Boletín de la Asociación de Geógrafos Españoles, (47), 79-107. https://dialnet.unirioja.es/servlet/articulo?codigo=3093402

Lane, S. N., Landström, C., \& Whatmore, S. J. (2011). Imagining flood futures: risk assessment and management in practice. Philosophical Transactions of the Royal Society of London A: Mathematical, Physical \& Engineering Sciences, 369(1942), 1784-1806. https://doi. org/10.1098/rsta.2010.0346

Nájera Ibáñez, A. \& Espejo Gil, F. (2017). Experiencia siniestral del cCs en relación con el riesgo de inundación: gestión y prevención. En Taller sobre inundaciones y cambio climático. Ministerio de Agricultura y Pesca, Alimentación y Medio Ambiente, Madrid, España.

Olcina Cantos, J. (2012). De los mapas de zonas afectadas a las cartografías de riesgo de inundación en España. Anales de Geografía de la Universidad Complutense, 32(1), $91-$ 131. https://doi.org/10.5209/rev AGUC.2012.v32.n1.39310

Parker, D. J. (1995). Floodplain development policy in England and Wales. Applied Geography, 15(4), 341-363. https://doi.org/10.1016/0143-6228(95)00016-W

Real Decreto 9/2008, de 11 de enero, por el que se modifica el Reglamento del Dominio Público Hidráulico, aprobado por el Real Decreto 849/1986, de 11 de abril. Boletín Oficial del Estado, Madrid, España. BOE-A-2008-755.

Real Decreto 903/2010, de 9 de julio, de evaluación y gestión de riesgos de inundación. Boletín Oficial del Estado, Madrid, Espańa. воE-A-2010-11184.

White, G. F. (1958). Changes in urban occupancy of flood plains in the U.S., vol. 57. Chicago, IL: University of Chicago. 